cirrhosis and decompensation at presentation as covariates) showed similar outcomes in both groups. Seventeen of the 23 patients continued MMF to end of follow-up and 6 discontinued MMF (lack of efficacy 4, successfully restarting of AZA 1 and cause unknown 1).

Conclusions Patients switching to MMF because of AZA intolerance do not differ from those continuing AZA in regard to: (a) normalisation of serum ALT (b) necro-inflammatory and fibrosis scores on follow-up biopsy and (c) 5- and 10 year mortality. This supports use of $\mathrm{MMF}$ as a steroid sparing agent in AZA intolerant patients.

\section{PTH-095 MINIMISING NON-HEPATIC COMPLICATIONS FOLLOWING LIVER TRANSPLANT: EVALUATION OF PRACTICE FROM A SINGLE REFERRAL CENTRE}

James Hawken*, Ben Hudson, Talal Valliani. North Bristol NHS Trust, Bristol, UK

\subsection{6/gutjnl-2018-BSGAbstracts.251}

Introduction Non-hepatic complications of liver transplant are common and associated with significant morbidity and mortality. The American Association for the Study of Liver Diseases (AASLD) practice guideline on the long-term management of liver transplant recipients aims to assist with modifying the risk of these complications. There is currently no equivalent European guideline. We analysed clinical records from a large UK centre to ascertain whether post-transplant care was comparable to the AASLD standards of care.

Methods Consecutive patients who had been transplanted at two UK centres following referral from a single UK centre between 1988 and 2016 were analysed retrospectively. All clinical documentation and test Results over a 12 month period were analysed. Outcome measures were aligned with AASLD guidelines, including; screening for diabetes, chronic kidney disease, hypertension, dyslipidaemia and osteopenia.

Results 48 patients ( 29 female/19 male) were included in analysis. Mean age was 57 (SD 13.4). Median time since transplantation was six years (IQR 3-13). 10/17 (58.8\%) diabetic patients met the recommendation of having their $\mathrm{HbA1c}$ measured in the preceding 3 months. Of non-diabetic patients, $15 / 29(51.7 \%)$ underwent annual fasting glucose (or HbA1c) during the study period. $48 / 48(100 \%)$ of patients had evidence of renal function monitoring within the last 12 months. Yearly urine albumin-creatinine ratio testing was performed in $10 / 48$ (20.8\%). Blood pressure was measured in 13/48 (27\%) of patients. Of those, $7 / 13(53.8 \%)$ had a satisfactory blood pressure of $<130 / 80 \mathrm{mmHg}$. The recommendation of annual blood lipid measurement took place in 30/48 (62.5\%) and annual Vitamin D in 20/48 (41.7\%). 8/18 (44.4\%) of patients transplanted between 2005 and 2012 underwent a Dual Energy X-ray Absorptiometry (DEXA) scan within 5 years. 12/ $14(85.7 \%)$ of osteopenic patients were receiving the recommended calcium supplementation, while 2/14 (14.3\%) of them were receiving annual Bone Mineral Density testing.

Conclusion Liver transplant recipients in this study did not receive a consistent approach to screening for common nonhepatic complications. Although there was often evidence that these complications were appropriately considered, there was wide variability between patients. The level of monitoring for patients, who are high risk for cardiovascular and metabolic disease, was insufficient overall. Consideration should be made to adoption of models of care which provide standardised recommendations for patients in the post-transplant phase. This may lead to a more rigorous and robust approach to patients' long-term management, which in turn may reduce late morbidity and mortality.

\section{PTH-096 ANTIBIOTIC USE IN PRIMARY AND SECONDARY PROPHYLAXIS OF SPONTANEOUS BACTERIAL PERITONITIS FOR LIVER CIRRHOSIS PATIENTS}

Arif Hussenbux*, Lysander Gourbault, Victoria Blackwell, John Ryan, Jane Collier. John Raddliffe Hospital, Oxford, UK

\subsection{6/gutjnl-2018-BSGAbstracts.252}

Introduction Spontaneous Bacterial Peritonitis (SBP) is associated with $30 \%-50 \%$ mortality within 1 year and $70 \%$ chance of recurrence. EASL guidelines state prophylactic antibiotics should be given to patients with proven SBP (secondary prophylaxis) and patients with low total protein count $(<15 \mathrm{~g} / \mathrm{L})$ in ascitic fluid with no prior history of SBP (primary prophylaxis). We audited whether these guidelines were followed and the associated mortality risk.

Methods Data on all ascitic taps for patients with confirmed liver cirrhosis were collected retrospectively over a 12 month period.

SBP patients were assessed as to whether secondary prophylactic antibiotics were commenced. The same investigations were carried for bacterascites (BA) patients, including whether BA was treated. Previous ascitic taps were analysed for low total protein count $(<15 \mathrm{~g} / \mathrm{L})$.

Cirrhotic patients without SBP or BA had their ascitic total protein count measured. If less than $15 \mathrm{~g} / \mathrm{L}$ we assessed whether primary prophylactic antibiotics were started. We assessed mortality rate for all patients.

Results Data collection period was from 15/10/2016 to $15 / 10$ / 2017 yielding 860 ascitic taps. Of the 89 patients with liver cirrhosis; infection was identified in 33 patients; 16 patients with SBP and 17 patients with BA.

Gram negative organisms were identified in $4 / 7$ with SBP and $7 / 17$ with bacterascites.

Secondary prophylaxis was started in 10 of $16(62.5 \%)$ patients with SBP. Of the patients where secondary prophylaxis was not started 3 of 6 (50\%) died within 12 months. All 16 patients had a previous tap within 12 months (mean 3.4 months) with ascitic protein count less than $15 \mathrm{~g} / \mathrm{L}$. Primary prophylaxis was not started for any patients.

10 of $17(58 \%)$ BA patients received intravenous antibiotic treatment. From this group 6 of $10(60 \%)$ received secondary prophylaxis. 6 of $17(35 \%)$ patients died within 12 months and none of these patient commenced secondary prophylaxis. All 17 patients had a previous tap within 12 months (mean 4.2 month) with ascitic protein count less than $15 \mathrm{~g} / \mathrm{L}$.

Of the 56 patients without SBP or BA 33 (58.9\%) had an ascitic protein count of less than $15 \mathrm{~g} / \mathrm{L}$. No patients were started on primary prophylactic antibiotics. 6 of $33(18.1 \%)$ patients with low protein ascites subsequently developed SBP when reviewed prospectively. No patients with ascitic protein count greater than $15 \mathrm{~g} / \mathrm{L}$ have developed SBP or BA. 16 of $33(48.4 \%)$ patients with low protein ascites died over the next 12 months.

Conclusion 66 of 89 (74\%) patients had low protein ascites and 50\% (33 of 66) subsequently developed either SBP or BA within 12 months. This highlights the importance of primary prophylactic antibiotics for patients with low protein ascites in the prevention of SBP and BA. 